\title{
Paternity Leave: Traversing the Landscape as Trainee and Faculty
}

\author{
Ravi Shankar ${ }^{1}$ (B)
}

Received: 21 September 2020 / Accepted: 29 March 2021 / Published online: 7 April 2021

(C) Academic Psychiatry 2021

Navigating through the academic landscape for an early career psychiatrist can sometimes pose as striking a perfect balance between two opposing forces. One of establishing a sense of identity in a new work environment that would assist in academic growth and career development and the other balancing time with a growing family, continuing to engage in selfinterests that would support a sense of well-being and connectedness with family and personal development.

As child psychiatrists, the importance of positive parental involvement in a child's life is ingrained early in training. For a stronger parental bond, increased paternal involvement in infant care is encouraged [1]. Yet, when it comes to trainees and academic physicians who are males, non-birthing parents, adoptive parents, or same-sex parents, it does seem hypocritical that there are no robust guidelines to enable such trainees to take time off to be involved in the care of their infant or child. When my first child was born, I was still in training and got no "paternity leave." In fact, my daughter was born on a Saturday and the following Monday I was back at work. Making that choice of returning to work immediately came with significant guilt — of leaving a new mother with minimal support, and of not being able to spend quality time with a newborn. Personally, as a trainee, several factors played into my hesitation in requesting time off, such as - not wanting to be the trainee who cancels clinic or who ends up extending his training and consequently gets entangled in the procedural complications of an already complicated international medical graduate journey. Many times, just the act of asking for extended time off may invoke feelings of guilt-Will this extend my training? Will this come across as unprofessional? What additional responsibilities can I or should I take on to compensate for this time off I'm getting? What other reasons and explanations can I give to make a "more compelling reason" why I need the time off? But the most important of them all

Ravi Shankar

shankarr@health.missouri.edu

1 University of Missouri, Columbia, MO, USA was not having a work culture where there was modeling or encouragement to taking time off as a new father.

Eventually, I graduated from training and moved to take a faculty position. Pretty soon after, my wife and I were thinking of having a second child. The issue of being able to strike the right balance between my family and my career surfaced again. If I chose one, was I causing a disservice to the other? Would this cost my academic career as an early career academician? Would it cost my relationships? How do I strike the right balance without feeling guilty? Fortunately, at this time, I was working within an institution that permitted paid parental leave and had a supportive administration. Yet I still felt I was in the minority as a male asking for this extended time off since parenting responsibilities have by default been unfairly delegated to female partners, and therefore, spent months wondering how to approach this topic with my supervisor. Nevertheless, the conversation happened, and I was able to take the time off to transition into the role of a father of two, support my wife through a difficult recovery, and make a positive parenting impact on the lives of our children. This was made possible by a supportive work environment where steps were taken to ensure that my work was delegated appropriately.

Currently, I serve as program director for the Child and Adolescent Psychiatry Fellowship program and Psychiatry Clerkship Director for medical students at my institution. These roles have allowed me to hear from mentors, peers, and trainees about their struggles with work-life balance, especially when it comes to parenting. I often find myself empathizing deeply with what my trainees go through since I had been there myself. With the current pandemic situation forcing increasing numbers of children to switch to virtual learning, the roles, duties, and stressors of parenthood have increased manifold.

The recently released American Board of Medical Specialties (ABMS) Policy on Parental, Caregiver, and Medical Leave During Training [2] is, therefore, an important step towards providing better support to trainees as they transition into the role of a parent. This new policy permits a minimum of 6 weeks away from training for new parents 
without extending their training time as long as competency, as determined by the clinical competency committee, is established. The policy released by ABMS presents an opportunity for Graduate Medical Education leadership and departments to discuss ways to best support trainees and junior faculty members transitioning into parenthood. Within the fellowship program at our institution, for example, we permit additional parental leave beyond the time allotted to fellows, provided there is advanced notice of such a request. To compensate for the additional time off, innovative educational experiences are accommodated during these times that strike a balance between training requirements and personal goals without necessarily extending the duration of training. For example, engaging in an educational experience focused on normal development, parental attachment, and bonding, course development, research/teaching interests, etc. Since program culture can impact trainees' and junior faculty's decision to take time off, the role of senior leadership in offering a transparent, safe environment where the practice of these policies is encouraged, without judgment, will be just as important as the establishment of uniform guidelines [3].

ABMS is crucial to facilitate the establishment of more nationally standardized parental leave policies that are transparent and accommodating [4]. It would also be key to have continued dialog with key stakeholders such as the
Accreditation Council for Graduate Medical Education. The equal involvement of both parents in childcare may be a positive step in gender equity and wellness for all involved and contribute towards positive child development.

\section{Declarations}

Disclosures The author states that there is no conflict of interest.

\section{References}

1. Ortiz Worthington R, Feld LD, Volerman A. Supporting new physicians and new parents: a call to create a standard parental leave policy for residents. Acad Med. 2019;94:1654-7.

2. American Board of Medical Specialties. American Board of Medical Specialties policy on parental, caregiver and medical leave during training. Effective July 1, 2021. Available from: https://www.abms. org/wp-content/uploads/2020/11/parental-caregiver-and-medicalleave-during-training-policy.pdf. Accessed 19 March 2021.

3. Paladine HL, Wendling A, Phillips J. The importance of changing culture on parental leave. Acad Med. 2020;95:488-9.

4. Pendleton KM, Salles A. Maternity leave: not just a challenge for trainees. Acad Med. 2020;95:489.

Publisher's Note Springer Nature remains neutral with regard to jurisdictional claims in published maps and institutional affiliations. 